MODELING, IDENTIFICATION AND CONTROL, 2001, VOL. 22, NO. 1, 3-14

doi:10.4173/mic.2001.11

\title{
Stabilization of Stable Manifold of Upright Position of the Spherical Pendulum
}

\author{
H. LUDVIGSEN $\ddagger$ A. SHIRIAEV $\dagger$ and O. EGELAND $\dagger$
}

Keywords: passivity, stabilization of the upright position of the spherical pendulum

The stabilization problem of the upright position of the sherical pendulum is treated in detail. This problem is reduced to the stabilization of the stable manifold $\Omega_{\mathrm{st}}$ of the upright position of the unforced spherical pendulum. It is shown that for any smooth feedback control derived by the speed-gradient algorithm with the objective to stabilize $\Omega_{\mathrm{st}}$ the closed loop system has a limit cycle $\Gamma$, which does not belong to the desired attractor $\Omega_{\mathrm{st}}$. It is shown that $\Gamma$ is hyperbolic.

\section{Introduction}

This paper is devoted to the further investigation connected with the problem of the swinging up of the spherical pendulum. The controlled spherical pendulum is a passive nonlinear system with two equilibrium points. These points, in contrast to the equilibriums of the planar pendulum, are only relative equilibriums. The vector field of the unforced spherical pendulum does not have any linear approximations in these points. The last property does not depend on the parametrization and is caused by the topological properties of the sphere.

The unforced spherical pendulum has two independent first integrals (the total energy $H_{0}$ and the generalized moment $p_{0}$, corresponding to rotation in the horizontal plane) and is a completely integrable nonlinear system. The upright position belongs to the invariant subset of the phase space defined by $H_{0}=2 m g l$ and $p_{0}=0$, where $m$, $l$ are mass and length of the pendulum; $g$ is the acceleration of gravity. By proposition 2.1 this subset is a stable manifold $\Omega_{\mathrm{st}}$ of the upright position. Thus the most natural way to solve the upright stabilization problem is trying to stabilize by the speedgradient-energy algorithm, see [3,4], the compact set of the cylindrical phase space defined by $H_{0}=2 m g l, p_{0}=0$.

As theorem 3.1 in part (a) states, see also [8], for any smooth feedback control derived by the speed gradient algorithm with the objective to stabilize $\Omega_{\mathrm{st}}$, the closed loop system will always possess a cycle $\Gamma$, which does not belong to the desired attractor $\Omega_{\mathrm{st}}$. Following these arguments a natural questions arise: When is this cycle unstable? or When does this cycle correspond to the exceptional set of initial conditions, for which the trajectory of the closed loop system does not tend to $\Omega_{\mathrm{st}}$ ?

The present paper complements the results of [8] and answers these questions. It is shown that $\Gamma$ is always hyperbolic and, moreover, the dimensions of the stable and unstable manifolds of the cycle $\Gamma$ are defined, see theorem 3.1 part (c).

The arguments developed in the paper can be applied to attack a slightly more

$\dagger$ Department of Engineering Cybernetics, Norwegian University of Science and Technology, N-7034 Trondheim, Norway. E-mail: Anton.Shiriaev@itk.ntnu.no; Olav.Egeland@ itk.ntnu.no

‡Cybernetica AS, Leirfossr 28, No-7038 Trondheim. E-mail: Halgeir.Ludvigsen@ cybernetica.no 
general problem: to stabilize a given invariant set of the unforced spherical pendulum corresponding to $H_{0}=H^{*}, p_{0}=p^{*}$, where $H^{*} \geqslant 0, p^{*} \in \mathbf{R}^{1}$, see the results in this direction for the general nonlinear systems in $[4,5,6,7]$. As it is shown in proposition 2.4 the closed loop system, with any feedback control providing the strict passivity, will have at least one cycle outside the desired attractor for any constants $H^{*}, p^{*}$ mentioned above. But for the case $p^{*} \neq 0$ the number of these exceptional cycles cannot be found analytically. By this reason the main results cope only with $H^{*}=2 \mathrm{mgl}$, $p^{*}=0$, having in mind the possibility to extend them for $H^{*} \geqslant 0, p^{*}=0$.

The paper is organized as follows. In section 2 the important auxiliary results are collected. Section 3 contains the main results of the paper. The results of the computer simulation are presented in section 4 . All proofs are brought to section 5 and in section 6 some conclusions are given.

\section{Preliminaries and Problem Statement}

The motions of the controlled spherical pendulum are described by the EulerLagrange equations

$$
\begin{aligned}
\frac{d}{d t} \frac{\partial \mathscr{L}(q, \dot{q})}{\partial \dot{q}_{1}}-\frac{\partial \mathscr{L}(q, \dot{q})}{\partial q_{1}} & =g_{1}(q, \dot{q}) u_{1}, \\
\frac{d}{d t} \frac{\partial \mathscr{L}(q, \dot{q})}{\partial \dot{q}_{2}} & =g_{2}(q, \dot{q}) u_{2},
\end{aligned}
$$

where $q=\left(q_{1}, q_{2}\right), \dot{q}=\left(\dot{q}_{1}, \dot{q}_{2}\right)$ are the coordinates and velocities of the spherical pendulum; $\mathscr{L}(q, \dot{q})$ is the Lagrangian function

$$
\mathscr{L}(q, \dot{q})=\frac{1}{2} m l^{2}\left[\left(\dot{q}_{1}\right)^{2}+\left(\dot{q}_{2}\right)^{2} \sin ^{2} q_{1}\right]-m g l\left(1-\cos q_{1}\right) ;
$$

$m, l$ are mass and length of the pendulum, $g$ is the acceleration of gravity; $g_{1}(q, \dot{q})$, $g_{2}(q, \dot{q})$ are scalar smooth functions; $u_{1}, u_{2}$ are control functions; see Figure 1 .

It is convenient to choose as a phase space for the spherical pendulum the cylin-

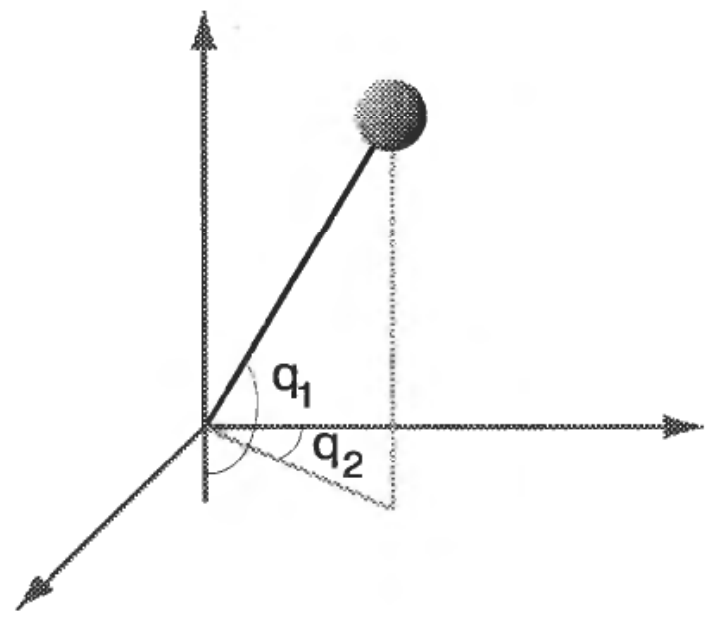

Figure 1. The spherical pendulum. 
drical space with a sphere of radius $l$ in the base, i.e. $0 \leqslant q_{1} \leqslant \pi, 0 \leqslant q_{2}<2 \pi$. The unforced system (1), (2) is completely integrable. Indeed, the functions

$$
\begin{aligned}
& H_{0}(q, \dot{q})=\frac{1}{2} m l^{2}\left[\left(\dot{q}_{1}\right)^{2}+\left(\dot{q}_{2}\right)^{2} \sin ^{2} q_{1}\right]+m g l\left(1-\cos q_{1}\right) \\
& p_{0}(q, \dot{q})=m l^{2}\left(\dot{q}_{2}\right) \sin ^{2} q_{1}
\end{aligned}
$$

are the independent first integrals of the unforced spherical pendulum with $H_{0}(q, \dot{q}) \geqslant 0, p_{0}(q, \dot{q}) \in \mathbf{R}^{1}$. Take the constants $H^{*} \geqslant 0, p^{*} \in \mathbf{R}^{1}$ and introduce the scalar function $V$ and the dummy output $y=\left(y_{1}, y_{2}\right)^{T}$ of the system (1), (2) as follows

$$
\begin{gathered}
V(q, \dot{q})=\frac{r_{1}}{2}\left[H_{0}(q, \dot{q})-H^{*}\right]^{2}+\frac{r_{2}}{2}\left[p_{0}-p^{*}\right]^{2} . \\
y_{1}=r_{1} \cdot \dot{q}_{1} \cdot g_{1}(q, \dot{q}) \cdot\left[H_{0}(q, \dot{q})-H^{*}\right], \\
y_{2}=g_{2}(q, \dot{q}) \cdot\left(r_{1} \cdot \dot{q}_{2} \cdot\left[H_{0}(q, \dot{q})-H^{*}\right]+r_{2} \cdot\left[p_{0}(q, \dot{q})-p^{*}\right]\right),
\end{gathered}
$$

where $r_{1}, r_{2}$ are some positive constants. The functions $V, y$ possess an important property: the controlled spherical pendulum (1), (2) with output function $y$ is a passive system with nonnegative storage function $V$, see definition 2.4 [1]. In particular, the derivative of the function $V$ along the solution of the system (1), (2) takes the form

$$
\frac{d}{d t} V(q, \dot{q})=y_{1} u_{1}+y_{2} u_{2} .
$$

The analysis of the qualitative behaviour of the closed loop system solutions with the feedback regulator formed by the speed-gradient algorithm is essentially based on the detailed investigation of the motions of the unforced spherical pendulum. The rest of this section is devoted to such important auxiliary results.

Proposition 2.1 ([8]) Denote $\Lambda_{p}$ as the maximal set of the trajectories $(q, \dot{q})$ of the unforced spherical pendulum such that the point $p$ of the cylindrical phase space is an $\omega$ limit point of any motion from $\Lambda_{p}$. Then

(1) the set $\Lambda_{(0,0,0,0)}=\{(0,0,0,0)\}$, i.e. there is no other trajectory except the point $(0,0,0,0)$, for which this equilibrium is its $\omega$-limit point;

(2) the set $\Lambda_{(\pi, 0,0,0)}$ has the form

$$
\Lambda_{(\pi, 0,0,0)}=\left\{\hat{q}_{1}(t), q_{2}, \dot{\hat{q}}_{1}(t), 0\right),
$$

where $q_{2}$ is any constant from $[0,2 \pi)$; the pair $\left.\hat{q}_{1}(t), \hat{\hat{q}}_{1}(t)\right)$ is a solution of the equation

$$
m l^{2} \cdot \ddot{\hat{q}}_{1}(t)=-m g l \cdot \sin \hat{q}_{1}(t)
$$

subjected to the constraint

$$
\left.\frac{1}{2} m l^{2} \dot{q}_{1}(t)\right)^{2}+m g l\left(1-\cos \hat{q}_{1}(t)\right)=2 m g l, \quad \forall t \geqslant 0 .
$$

Moreover, the upright position is a unique $\omega$-limit point of any trajectory from $\Lambda_{(\pi, 0,0,0)}$

Consider the motion $(q, \dot{q})$ of the unforced spherical pendulum with $\dot{q}_{2} \neq 0$. By proposition 2.1 this trajectory does not belong to the sets $\Lambda_{(0,0,0,0)}, \Lambda_{(\pi, 0,0,0)}$. Therefore the smooth function $q_{1}(t)$ is strictly bounded away from zero and $\pi$. By this reason the trajectory $(q, \dot{q})$ satisfies the equations 


$$
\begin{aligned}
& \ddot{q}_{1}=\sin q_{1} \cdot \cos q_{1} \cdot\left(\dot{q}_{2}\right)^{2}-\frac{g}{l} \cdot \sin q_{1}, \\
& \ddot{q}_{2}=-2 \cdot \frac{\cos q_{1}}{\sin q_{1}} \cdot \dot{q}_{1} \cdot \dot{q}_{2},
\end{aligned}
$$

which is well defined for all $t \geqslant 0$.

Theorem 2.2 Suppose that the smooth functions $g_{1}, g_{2}$ are not identically equal to zero along any solution of the unforced spherical pendulum. Let $(q, \dot{q})$ be any motion of the unforced spherical pendulum consistent with $y(t)=0$ for all $t \geqslant 0$, where $y$ is defined by (7.1), (7.2). Then only the following cases are possible:

(1) $H_{0}(q(t), \dot{q}(t))=H^{*}, p_{0}(q(t), \dot{q}(t))=p^{*}$;

(2) $(q(t), \dot{q}(t))=(\pi, 0,0,0)$ or $(q(t), \dot{q}(t))=(0,0,0,0), p^{*}=0$;

(3) $(\hat{q}(t), \hat{q}(t))=\left(q_{1}^{*}, q_{2}^{*} \cdot t \bmod 2 \pi, 0, q_{2}^{*}\right)$, where the constants $q_{1}^{*}, q_{2}^{*}$ satisfy the equation

$$
\left\{\begin{array}{r}
r_{1} \cdot q_{2}^{*} \cdot\left[H_{0}(\hat{q}, \hat{q})-H *\right]+r_{2} \cdot\left[p_{0}(\hat{q}, \hat{q})-p^{*}\right]=0 \\
\left(q_{2}^{*}\right)^{2} \cdot \cos q_{1}^{*}=\frac{g}{l}
\end{array}\right.
$$

Remark 2.3 The assumptions of theorem 2.2 imposed on the function $g_{1}, g_{2}$ are not restrictive. In particular, they are satisfied by $g_{1}=c_{1}, g_{2}=c_{2}$, where $c_{1}, c_{2}$ are any nonzero constants.

Proposition 2.4 (Existence of cycle) Let $p^{*} \in \mathbf{R}^{1}$ and $H^{*}, r_{1}, r_{2}$ be any positive constants, then equations (10) has at least one solution. If $p^{*}=0$ and $H^{*}, r_{1}, r_{2}$ any positive constants then equations (10) has exactly one solution. If, in addition, $H^{*}=2 m g l$ the solution is

$$
\begin{aligned}
\left(q_{1}^{*}\right)^{2} & =\frac{1}{2}\left[\left(3 \frac{g}{l}-2 \frac{r_{2}}{r_{1}}\right)+\sqrt{\left(3 \frac{g}{l}-2 \frac{r_{2}}{r_{1}}\right)^{2}+8 \frac{r_{2} g}{r_{1} l}}\right], \\
q_{1}^{*} & =\arccos \left(\frac{g}{l} \frac{1}{\left(q_{2}^{*}\right)^{2}}\right) .
\end{aligned}
$$

Remark 2.5 Strictly speaking there are two solutions $\left( \pm q_{2}^{*}\right)$, but they represent the same set in the phase space. When $p^{*} \neq 0$ this symmetri is lost, and there might be several solutions.

Remark 2.6 It follows from the proof of proposition 2.4 that for $p^{*} \neq 0$ the solvability of the equations (10) is equivalent to the solvability of some 6th order equation. Thus even the definition of the number of soultions of (10) with an arbitrary admissible parameters $H^{*} \geqslant 0, p^{*} \in \mathbf{R}^{1}$ encounters substantial computational problems and cannot be solved analytically.

\section{Main Results}

Let $\phi_{1}(z), \phi_{2}(z)$ be any scalar smooth functions satisfying the inequalities

$$
\varepsilon_{1}|z|^{2} \leqslant\left\{z \phi_{1}(z), z \phi_{2}(z)\right\} \leqslant \varepsilon_{2}|z|^{2}, \quad \forall z \in \mathbf{R}^{1},
$$

where $\varepsilon_{2} \geqslant \varepsilon_{1}>0$, and take the regulator of the form 


$$
u_{1}=-\phi_{1}\left(y_{1}\right), \quad u_{2}=-\phi_{2}\left(y_{2}\right) .
$$

The following theorem reflects the qualitative behaviour of the solutions of the closed loop system (1), (2), (12) with the objective to stabilize the stable manifold of the upright position of the unforced spherical pendulum, which by proposition 2.1 coincides with the compact set $V_{0}=\left\{(q, \dot{q}): H_{0}(q, \dot{q})=2 m g l, p_{0}(q, \dot{q})=0\right\}$.

Theorem 3.1 Let $H^{*}=2 m g l, p^{*}=0$ and $r_{1}, r_{2}$ be any positive constants, and the functions $g_{1}, g_{2}$ not identically equal to zero along any solution of the unforced spherical pendulum. Let $\phi_{1}, \phi_{2}$ be any scalar smooth functions satisfying the inequalities (11). Define the regulator by relation (12). Then

(a) for any solution $(q, \dot{q})$ of the closed loop system, which does not coincide with the equilibrium points $(0,0,0,0),(\pi, 0,0,0)$, only the following alternatives are possible:

(1) $\lim _{t \rightarrow+\infty} H_{0}(q(t), \dot{q}(t))=H^{*}, \lim _{t \rightarrow+\infty} p_{0}(q(t), \dot{q}(t))=0$

(2) the trajectory $(q(t), \dot{q}(t))$ tends as $t \rightarrow+\infty$ to the unique for any $r_{1}>0, r_{2}>0$ cycle $\Gamma$ of the unforced pendulum defined by motion $\left(q_{1}^{*}, q_{2}^{*} \cdot t \bmod 2 \pi, 0, q_{2}^{*}\right)$, where the constants $q_{1}^{*}, q_{2}^{*}$ satisfy equation (10),

(b) for any solution $(q(t), \dot{q}(t))$ of the closed loop system (1), (2), (12), which corresponds to the case $(a 1)$, the unstable equilibrium point $(q, \dot{q})=(\pi, 0,0,0)$ of the unforced spherical pendulum is a relative $\omega$-limit point, i.e. $\forall \varepsilon>0$ there exists a time sequence $\left\{T_{n}\right\}_{n=1}^{+\infty}, T_{n} \rightarrow+\infty$, such that $\left|q_{1}\left(T_{n}\right)-\pi\right|<\varepsilon,\left|\dot{q}_{1}\left(T_{n}\right)\right|<\varepsilon$.

(c) If the smooth functions $g_{1}, g_{2}$ are constants on $\Gamma$, then for any functions $\phi_{1}, \phi_{2}$, satisfying (11), the limit cycle $\Gamma$ is unstable. Moreover, the dimension of the stable manifold of $\Gamma$ is equal to 2 and the dimension of the unstable manifold of $\Gamma$ is equal to 3 .

Remark 3.2 Theorem 3.1 does not guarantee that even in the best case, when the solution $(q, \dot{q})$ of the closed loop system (1), (2), (12) tends to the set $V_{0}=\{(q, \dot{q})$ : $\left.H_{0}(q, \dot{q})=2 m g l, p_{0}(q, \dot{q})=0\right\}$, the trajectory $(q, \dot{q})$ will be in some relative neighbourhood of the upper equilibrium point for all sufficiently large moments of time. But this trajectory will be an infinite number of times in any relative neighbourhood of the upright position, see also the results of the computer simulations. Moreover, it is possible to show that the $\omega$-limit set of the closed loop system (1), (2), (12) is always larger than just the upright position, see [9].

Remark 3.3 The functions $g_{1}, g_{2}$ will be constants on $\Gamma$ if, for example, they do not depend on $q_{2}$, i.e. $g_{i}\left(q_{1}, q_{2}, \dot{q}_{1}, \dot{q}_{2}\right)=g_{i}\left(q_{1}, \dot{q}_{1}, \dot{q}_{2}\right), i=1,2$.

Remark 3.4 Thus the detailed analysis of the closed loop system (1), (2) with the feedback control (12) derived from the standard speed-gradient algorithm, shows that for almost all initial conditions the solution of the closed loop system will tend to the desired compact attractor, stable manifold of the upright position. Moreover, the set of the exceptional initial conditions, corresponding to the trajectories with undesirable behaviour (winding to the limit cycle $\Gamma$ ), is only a 2-dimensional smooth manifold.

Remark 3.5 It is worth to recall that for an autonomous system the sum of the dimensions of the stable and unstable manifolds of the cycle is equal to $n+1$, where $n$ is the dimension of the phase space. The phase space of the spherical pendulum equals to 4 , hence the sum of the dimensions of the stable and unstable manifolds of $\Gamma$ is 5 . 
The proof of theorem 3.1 in parts (a) and (b) can be found in [8]. The main contribution of the paper is concentrated in part (c) of theorem 3.1, which states the unstability of $\Gamma$. The proof of this statement is technical and consists of several standard steps, which are formulated in the form of the separated propositions.

Proposition 3.6 Let $H^{*}=2 m g l, p^{*}=0$ and $\phi_{1}, \phi_{2}$ be any smooth functions. Then the linear approximation of the closed loop system (1), (2), (12) around the cycle $\Gamma=\left(q_{1}^{*}, q_{2}^{*} \cdot t \bmod 2 \pi, 0, q_{2}^{*}\right)$, where $q_{1}^{*}, q_{2}^{*}$ are defined by $(10)$, has the form

$$
\frac{d}{d t}\left[\begin{array}{l}
x_{1} \\
x_{2} \\
x_{3} \\
x_{4}
\end{array}\right]=\left[\begin{array}{cccc}
0 & 0 & 1 & 0 \\
0 & 0 & 0 & 1 \\
\alpha_{1} & 0 & \alpha_{2} & \alpha_{3} \\
\beta_{1} & 0 & \beta_{2} & \beta_{3}
\end{array}\right]\left[\begin{array}{l}
x_{1} \\
x_{2} \\
x_{3} \\
x_{4}
\end{array}\right]
$$

where

$$
\begin{aligned}
& \alpha_{1}=-\left(q_{2}^{*}\right)^{2} \cdot \sin ^{2} q_{1}^{*}, \\
& \alpha_{2}=-g_{1}^{2}\left(q_{1}^{*}, q_{2}^{*} \cdot t \bmod 2 \pi, 0, q_{2}^{*}\right) \cdot \dot{\phi}_{1}(0) \cdot r_{1} \cdot\left[\frac{\left(q_{2}^{*}\right)^{2} \cdot \sin ^{2} q_{1}^{*}}{2}-\frac{g}{l}\left(1+\cos q_{1}^{*}\right)\right], \\
& \alpha_{3}=2 \cdot q_{2}^{*} \cdot \sin q_{1}^{*} \cdot \cos q_{1}^{*}, \\
& \beta_{1}=-\frac{g_{2}^{2}\left(q_{1}^{*}, q_{2}^{*} \cdot t \bmod 2 \pi, 0, q_{2}^{*}\right)}{\sin q_{1}^{*}} \cdot \dot{\phi}_{2}(0) \cdot 2 \cdot q_{2}^{*} \cdot\left[r_{1} \cdot \frac{g}{l}+r_{2} \cdot \cos q_{1}^{*}\right], \\
& \beta_{2}=-2 \cdot q_{2}^{*} \cdot \frac{\cos q_{1}^{*}}{\sin q_{1}^{*}}, \\
& \beta_{3}=-g_{2}^{2}\left(q_{1}^{*}, q_{2}^{*} \cdot t \bmod 2 \pi, 0, q_{2}^{*}\right) \cdot \dot{\phi}_{2}(0) \cdot r_{1} \cdot\left(q_{2}^{*}\right)^{2} .
\end{aligned}
$$

The assumption that the functions $g_{1}, g_{2}$ are constant on $\Gamma$ results in that the linear system (13) with periodic coefficients becomes time-invariant. This simplifies the stability analysis of the cycle $\Gamma$. Indeed, it is sufficient only to define the eigenvalues of the constant matrix involved in (13).

Proposition 3.7 The characteristic polynomial $p(\lambda)$ of the linear system $(13)$ has the form

$$
p(\lambda)=\lambda \cdot\left(\lambda^{3}+\gamma_{1} \cdot \lambda^{2}+\gamma_{2} \cdot \lambda+\gamma_{3}\right)
$$

where

$$
\begin{aligned}
& \gamma_{1}=g_{2}^{2} \cdot \dot{\phi}_{2}(0) \cdot r_{1} \cdot\left(q_{2}^{*}\right)_{1}^{2} g_{1}-2 \cdot \dot{\phi}_{1}(0) \cdot r_{2} \cdot \sin ^{2} q_{1}^{*}, \\
& \gamma_{2}=\left(q_{2}^{*}\right)^{2} \cdot\left[1+3 \cdot \cos ^{2} q_{1}^{*}-\left(g_{1} \cdot g_{2}\right)^{2} \cdot \dot{\phi}_{1}(0) \cdot \dot{\phi}_{2}(0) \cdot r_{1} \cdot r_{2} \cdot \sin ^{2} q_{1}^{*}\right] \text {, } \\
& \gamma_{3}=g_{2}^{2} \cdot \dot{\phi}_{2}(0) \cdot\left(q_{2}^{*}\right)^{2} \cdot\left[r_{1} \cdot\left(q_{2}^{*}\right)^{2} \cdot\left(1+3 \cdot \cos ^{2} q_{1}^{*}\right)+4 \cdot r_{2} \cdot \cos ^{2} q_{1}^{*}\right] \text {, }
\end{aligned}
$$

Proposition 3.8 Suppose that the smooth functions $\phi_{1}, \phi_{2}$ satisfy (11) and $r_{1}, r_{2}$ are positive constants. Then the set $\mathscr{R}$ of the roots of the polynomial $p(\lambda)$, defined by (14), is

$$
\mathscr{R}=\left\{0,-\lambda_{0}, \lambda_{1}, \bar{\lambda}_{1}\right\},
$$

where $\lambda_{0}>0$ and $\operatorname{Re} \lambda_{1}>0$. 

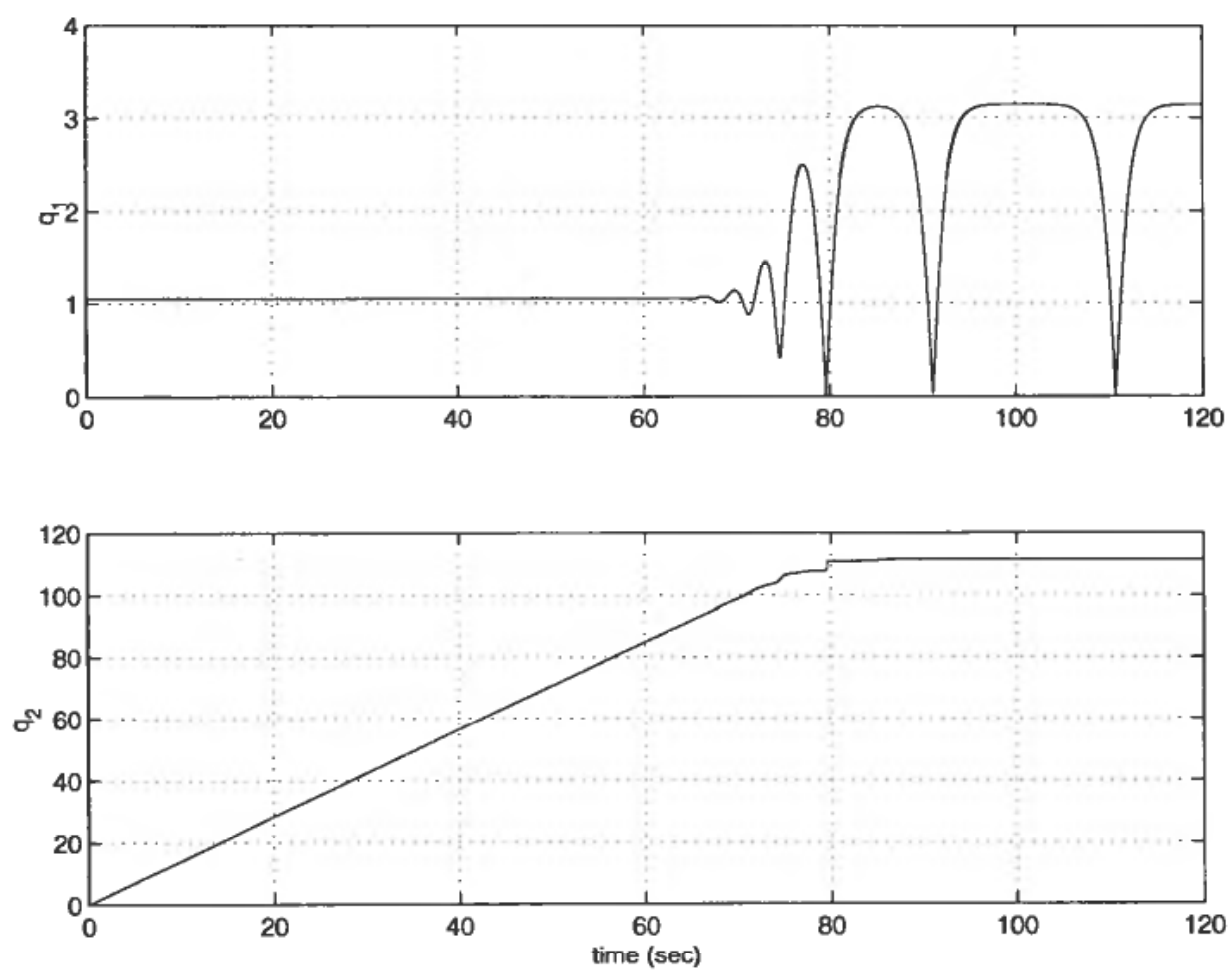

Figure 2. System states with initial conditions on the cycle.

\section{Computer simulations}

Simulating the unstable cycle is difficult. Computational roundoff errors sum up very fast, and the system will leave the set. An example of this behaviour is shown in Figures 2 and 3, where after 80 seconds the controller has taken the system to the stable energy level. In this simulation (the simulations were made in Matlab 5/ SIMULINK, a trademark of The MathWorks, Inc.) the parameters were:

$$
m=1, l=g=9.81, r_{1}=r_{2}=1, g_{1}=g_{2}=1, \phi(y)=y
$$

Using proposition 2.4 , we find that the cycle is given by $(\hat{q}(t), \dot{q}(\mathrm{t}))=$ $(\pi / 3,0.5 \cdot t, 0,0.5)$. The initial values used in the simulation were $(q(0), \dot{q}(0))=$ $(\pi / 3,0,0,0.5)$. Figure 5 shows how an error $q_{1}(0)=\pi / 3-\varepsilon$ affects the response. The error used in the simulation were $\varepsilon=0.000001$.

Notice that $H_{0}$ and $p_{0}$ are not going monotonically to the final values.

\section{Appendix}

Proof of theorem 2.2 It is easy to see that if the trajectory $(q, \dot{q})$ of the unforced spherical pendulum is one of the equilibrium points or along this motion $H_{0}(q, \dot{q})=H^{*}$ and $p_{0}(q, \dot{q})=p^{*}$, then this motion is consistent with $y_{1}(t)=0, y_{2}(t)=0$ for all $t \geqslant 0$, where $y_{1}, y_{2}$ are defined by (7.1), (7.2).

Suppose that $(q, \dot{q})$ is some different trajectory of the unforced spherical pendulum which satisfies $y_{1}(t)=0, y_{2}(t)=0$ for all $t \geqslant 0$. The functions $H_{0}(q, \dot{q}), p_{0}(q, \dot{q})$ are the conserved quantities of the unforced spherical pendulum, hence they are equal to the constants $H_{0}(q(0), \dot{q}(0)), p_{0}(q(0), \dot{q}(0))$ for all $t \geqslant 0$. 

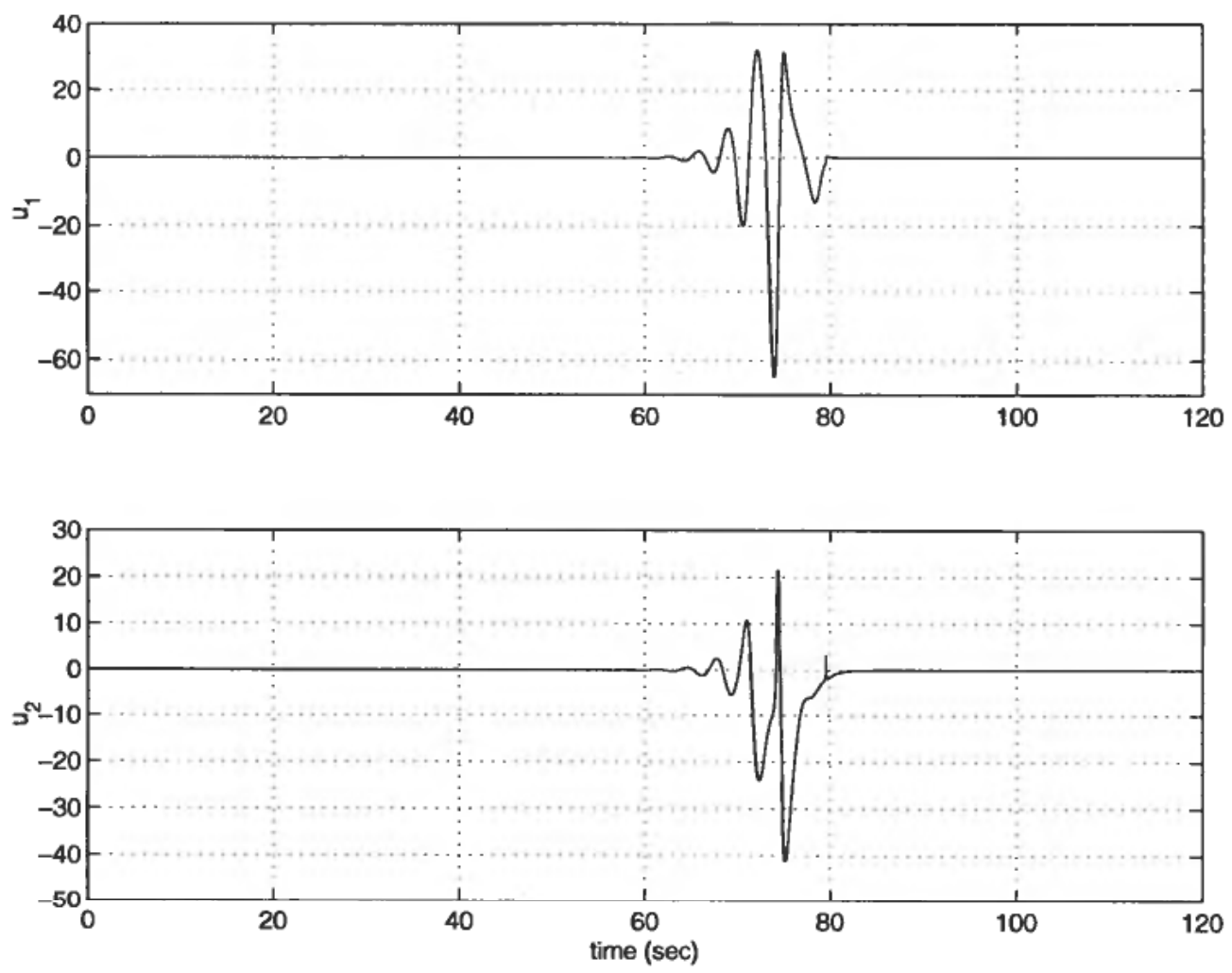

Figure 3. Control signal with initial conditions on the cycle.

Let $p_{0}(q(0), \dot{q}(0)) \neq p^{*}$ and $H_{0}(q(0), \dot{q}(0))=H^{*}$, then the relation $y_{2}(t)=0, t \geqslant 0$, implies, see (7.2), that $g_{2}(q(t), \dot{q}(t)) \cdot\left[p_{0}(q(t), \dot{q}(t))\right]=0, \mathrm{t} \geqslant 0$. But assumption $g_{2}(q, \dot{q})$ is not identically equal to zero along any motion of the unforced spherical pendulum. Thus $p_{0}(q(0), \dot{q}(0))=p^{*}$.

Let $H_{0}(q(0), \dot{q}(0)) \neq H^{*}$, then the relation $y_{1}(t)=0, t \geqslant 0$, implies, see (7.2), that $\dot{q}(t) \cdot g_{1}(q(t), \dot{q}(t))=0, t \geqslant 0$. By assumption $g_{1}(q, \dot{q})$ is not identically equal to zero along any motion of the unforced spherical pendulum, then $\dot{q}(t)=0, t \geqslant 0$, i.e. $q_{1}(t) \equiv$ const. By virtue (8) the last relation means that

$$
\sin q_{1} \cdot\left(\cos q_{1} \cdot\left(\dot{q}_{2}\right)^{2}-\frac{g}{l}\right)=0
$$

By our temporary assumption $q_{1} \neq 0, \pi$, hence the value of $\dot{q}_{2}(t)$ is uniquely up to the sign defined and $\dot{q}_{2}(t)$ is a constant.

Thus if $(q, \dot{q})$ is any motion of the unforced spherical pendulum consistent with $y_{1}(t)=0, y_{2}(t)=0, t \geqslant 0$, and $(q, \dot{q})$ does not satisfy the conditions (1) and (2) of theorem 2.2, then this motion has the form $q_{1}(t)=q_{1}^{*}, q_{2}(t)=q_{2}^{*} \cdot t$, where the constants $q_{1}^{*}, q_{2}^{*}$ are the solution of the equations (10).

Proof of proposition 2.4 Substitute $H_{0}$ and $p_{0}$ from (4) and (5), and use ( $\hat{q}(t)$, $\hat{\hat{q}}(t))=\left(q_{1}, q_{2} \cdot t, 0, q_{2}\right)$ to write $(10)$ as 

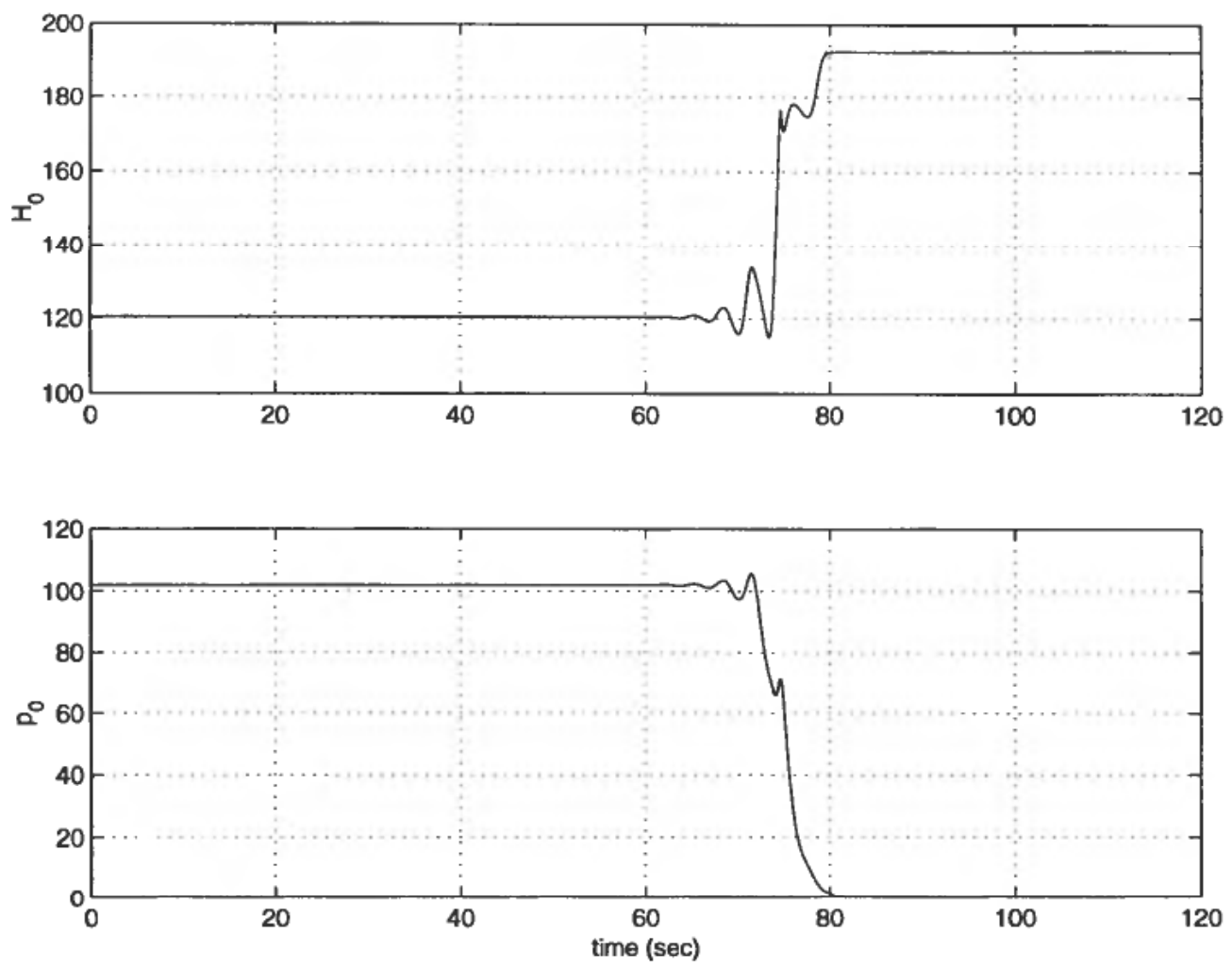

Figure 4. Integral values with initial conditions on the cycle.

$$
\begin{aligned}
r_{1} q_{2}\left(\frac{1}{2} m l^{2} q_{2}^{2}\left(1-\cos ^{2} q_{1}\right)+m g l\left(1-\cos q_{1}\right)-H^{*}\right) & \\
+r_{2}\left(m l^{2} q_{2}\left(1-\cos ^{2} q_{1}\right)-p^{*}\right) & =0 \\
\cos q_{1} & =\frac{g}{l} \frac{1}{q_{2}^{2}}
\end{aligned}
$$

Substituting for $\cos q_{1}$, multiplying and collecting terms gives

$$
f\left(q_{2}\right)=\frac{r_{1}}{2} m l^{2} q_{2}^{6}+\left(r_{2} m l^{2}+r_{1} m g l-r_{1} H^{*}\right) q_{2}^{4}-r_{2} p^{*} q_{2}^{3}-\frac{3}{2} m g^{2} q_{2}^{2}-r_{2} m g^{2}=0
$$

In order to have a solution for $q_{1}, \cos q_{1}<1 \Leftrightarrow q_{2}^{2}>(g / l)$. Evaluating $f\left(q_{2}\right)$ in $\left(q_{2}^{*}\right)^{2}=(g / l)$

$$
f\left(q_{2}^{*}\right)=-r_{1} H^{*} \frac{g^{2}}{l^{2}}-r_{2} p^{*}\left(q_{2}^{*}\right)^{3}=0
$$

Noting that $f(\infty)>0$, there is at least one solution if $f\left(q_{2}^{*}\right)<0$. If $p^{*}>0$ the solution is with $q_{2}>+\operatorname{sqrt}(g / l)$, and for $p^{*}<0, q_{2}<-\operatorname{sqrt}(g / l)$.

Let

$$
p^{*}=0, \lambda=q_{2}^{2}, \tilde{r}=\frac{r_{2}}{r_{1}}, \tilde{g}=\frac{g}{l}, \tilde{H}=\frac{H^{*}}{m l^{2}}
$$



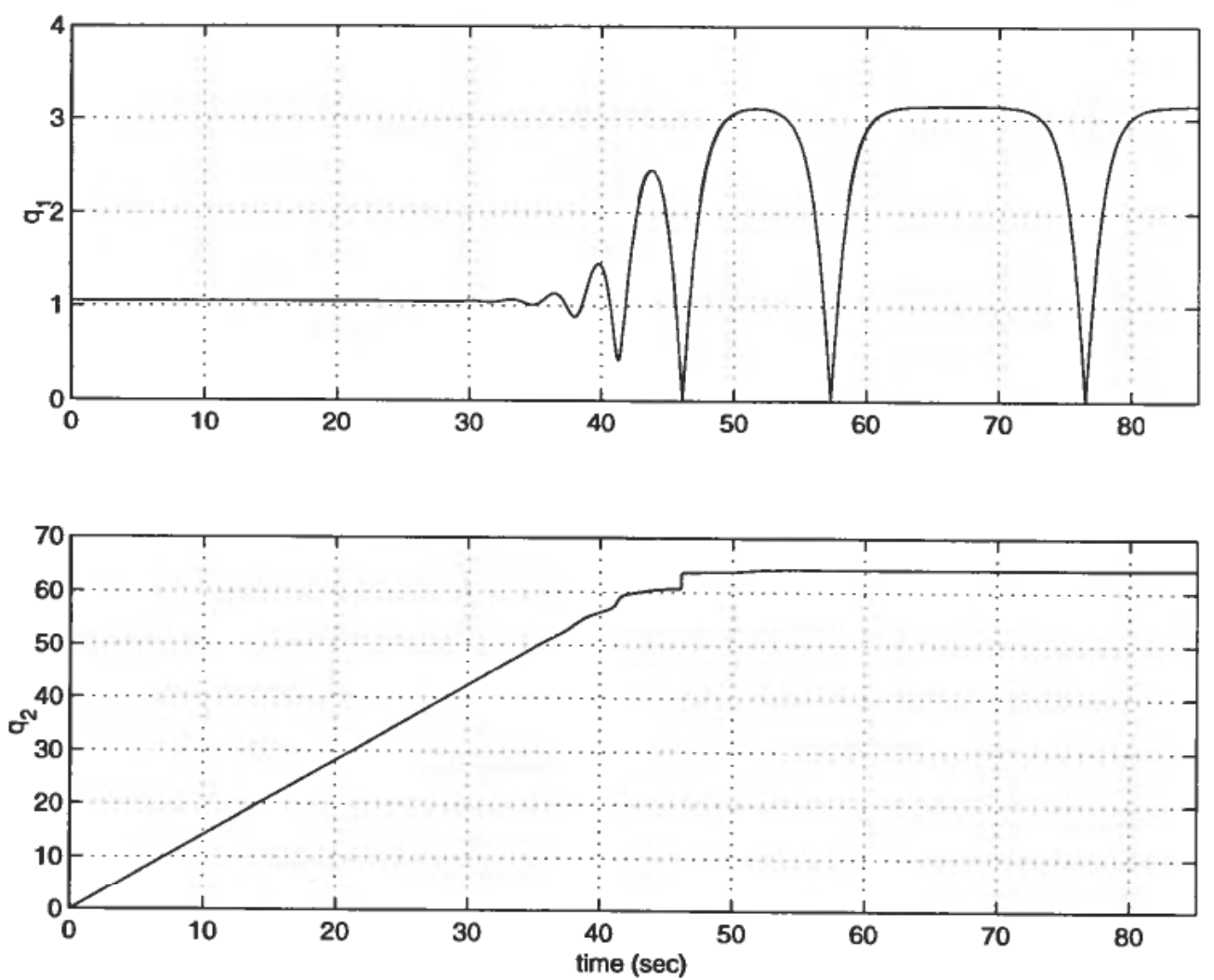

Figure 5. System states with error in the initial condition.

and rewrite the equation in these variables as

$$
f(\lambda)=\lambda^{3}+2(\tilde{r}+\sim g-\tilde{H}) \lambda^{2}-3 \tilde{g}^{2} \lambda-2 \tilde{r} \tilde{g}^{2}=0
$$

The requirement for a solution is now that $\lambda>\tilde{g}$. Since $f(\tilde{g})=-2 \tilde{H} \tilde{g}^{2}<0, f(\infty)>0$ there exists at least one solution for $\lambda$. Given a general third order polynomial

$$
\begin{aligned}
\left(\lambda-\lambda_{1}\right)\left(\lambda-\lambda_{2}\right)\left(\lambda-\lambda_{3}\right)= & \lambda^{3}-\left(\lambda_{1}+\lambda_{2}+\lambda_{3}\right) \lambda^{2}+\left(\lambda_{1} \lambda_{2}+\lambda_{1} \lambda_{3}+\lambda_{2} \lambda_{3}\right) \lambda_{\lambda_{1}} \lambda_{2} \lambda_{3} \\
& \left(\lambda_{1}, \lambda_{2}, \lambda_{3}>0\right) .
\end{aligned}
$$

Comparing with $f(\lambda)$ and noting that $f(0)<0$, the posibilities are that there is exactly one positive (real) solution, or all three solutions have positive real part (at least one real). The linear term in $f(\lambda)$ is negative, and rules out the last posibility. For $H^{*}=2 m g l \Leftrightarrow H=2 \tilde{g}$, one solution for the third order equation is $\lambda=-\tilde{g}$ and it reduses to

$$
f(\lambda)=(\lambda+\tilde{g}) \cdot\left(\lambda^{2}+(2 \tilde{r}-3 \tilde{g}) \lambda-2 \tilde{r} \tilde{g}\right)
$$

The positive root for the second order equation is

$$
\lambda=\frac{1}{2}\left[(3 \tilde{g}-2 \tilde{r})+\sqrt{(3 \tilde{g}-2 \tilde{r})^{2}+8 \tilde{r} \tilde{g}}\right]
$$

This proves proposition 2.4 . 
Proof of proposition 3.6 Let $\phi_{1}, \phi_{2}$ be any smooth scalar functions and let the feedback control be defined by (12). Then by smoothness and implicit function theorem there exists sufficiently small neighbourhood of the cycle $\Gamma$ such that for any solution of the closed loop system (1), (2), (12) with initial conditions from this neighbourhood will be strictly bounded away the download and upright equilibriums within the time interval $[0,2 \pi]$. Hence any solution of the closed loop system with such choosen initial condition will be well defined on $[0,2 \pi]$ by the equations

$$
\frac{d}{d t}\left[\begin{array}{l}
q_{1} \\
q_{2} \\
\dot{q}_{1} \\
\dot{q}_{2}
\end{array}\right]=\left[\begin{array}{l}
\dot{q}_{1} \\
\dot{q}_{2} \\
\sin q_{1} \cdot \cos q_{1} \cdot\left(\dot{q}_{2}\right)^{2}-\frac{g}{l} \cdot \sin q_{1}-\frac{g_{1}(q, \dot{q})}{m l^{2}} \cdot \phi_{1}\left(y_{1}\right) \\
-2 \cdot \frac{\cos q_{1}}{\sin q_{1}}-\frac{g_{2}(q, \dot{q})}{m l^{2} \cdot \sin ^{2} q_{1}} \cdot \phi_{2}\left(y_{2}\right)
\end{array}\right]=F(q, \dot{q}),
$$

where $y_{1}, y_{2}$ are defined by (7.1), (7.2). The linearization of the system (16) about the cycle $\Gamma$ is a linear $2 \pi$-periodic system

$$
\frac{d}{d t} x(t)=\frac{\partial F\left(q_{1}^{*}, q_{2}^{*} \cdot t \bmod 2 \pi, 0, q_{2}^{*}\right)}{\partial(q, \dot{q})} x(t)=A(t) x(t), \quad A(t)=A(2 \pi+t) .
$$

The straightforward computations lead to the system (13) with the coefficients mentioned in proposition 3.6.

Proof of proposition 3.7 Follows from the straightforward computations of the coefficients of the characteristic polynomial $p(\lambda)$.

Proof of proposition 3.8 In the origin the polynomial $p_{1}(\lambda)=p(\lambda) / \lambda$ is equal to $\gamma_{3}$ and, hence, positive. Then $p_{1}(\lambda)$ has at least one negative real root, i.e.

$$
p_{1}(\lambda)=\left(\lambda+\lambda_{0}\right) \cdot\left(\lambda^{2}+\rho_{1} \cdot \lambda+\rho_{2}\right),
$$

Where $\lambda_{0}, \rho_{1}, \rho_{2}$ are real constants, $\lambda_{0}>0, \rho_{2}>0$. Suppose temporarily that $p_{1}(\lambda)$ is a stable polynomial. This assumption implies that the coefficients $\gamma_{1}, \gamma_{2}, \gamma_{3}$, see (14), satisfy the following conditions

$$
\gamma_{1}>0, \quad \gamma_{2}>0, \quad \gamma_{3}>0, \quad \gamma_{1} \cdot \gamma_{2}-\gamma_{3}>0 .
$$

Calculating the value of $\gamma_{1} \cdot \gamma_{2}-\gamma_{3}$, we have

$$
\gamma_{1} \cdot \gamma_{2}-\gamma_{3}=-\alpha \cdot \beta \cdot \gamma_{1}-\beta \cdot \sin ^{2} q_{1}^{*} \cdot\left(1+3 \cdot \cos ^{2} q_{1}^{*}\right)-\frac{r_{1}}{r_{2}} \cdot 4 \cdot \alpha \cdot \cos ^{2} q_{1}^{*}<0,
$$

where $\alpha=\left(g_{2}\right)^{2} \cdot \dot{\phi}_{2}(0) \cdot r_{1}>0, \beta=\left(g_{1}\right)^{2} \cdot \dot{\phi}_{1}(0) \cdot r_{2}>0$. This shows that $p_{1}(\lambda)$ cannot be stable polynomial. The same arguments show that $p_{1}(\lambda)$ cannot have pure imaginary roots. Indeed, in this case $p_{1}(\lambda)$ should have the form

$$
p_{1}(\lambda)=\left(\lambda+\lambda_{0}\right) \cdot\left(\lambda^{2}+\rho_{2}\right),
$$

and, in particular, $\gamma_{1} \cdot \gamma_{2}-\gamma_{3}=0$. But this contradicts with (17). Therefore,

$$
p_{1}(\lambda)=\left(\lambda+\lambda_{0}\right) \cdot\left(\lambda-\lambda_{1}\right) \cdot\left(\lambda-\bar{\lambda}_{1}\right)
$$

and $\operatorname{Re} \lambda_{1}>0$ 


\section{Conclusions}

The paper deals with the stabilization of the stable manifold $\Omega_{\mathrm{st}}$ of the upright position of the unforced spherical pendulum. This spesial problem is naturally arised from the problem of the stabilization of the upright position of the spherical pendulum, see [8]. It was shown that, for any smooth feedback control derived from the speed gradient method with the objective to render the compact set $\Omega_{\mathrm{st}}$ being attractive, the closed loop system has a cycle $\Gamma$, which lies outside $\Omega_{\text {st }}$.

It was shown, and this is the main contribution of the paper, that the cycle $\Gamma$ is hyperbolic. Moreover, the dimensions of the stable and unstable manifolds of $\Gamma$ were obtained.

\section{References}

[1] Byrnes, C. I., Isidori, A. and Willems, J. C. (1991). Passivity, feedback equivalence and the global stabilization of minimum phase nonlinear systems, Transactions on $A C$, Vol. 36, N 11, pp. 1228-1240.

[2] FradKov, A. L. (1990). Adaptive control of complex systems. Moscow: Nauka. (Russian)

[3] FradKov, A .L. (1996). Swinging control of nonlinear oscillations, Internat. J. of Control, Vol. 64, N 6, pp. 1189-1202.

[4] Fradkov, A. L. Makarov, I. A., Shiriaev, A. S. and Tompchina, O. P. (1997). Control of oscillations in Hamiltonian systems, Proceedings of 4th European Control Conference, Brussels.

[5] Shiriaev, A. S. and Fradkov, A. L. (1998). Stabilization of invariant set of nonlinear systems. In: Nonlinear dynamical systems. St. Petersburg: St. Petersburg State University. (Russian).

[6] Shiriaev, A. S. (1998). The notion of $V$-detectability and stabilization of invariant set of nonlinear systems, Proceedings of 37th Conference on Decision and Control, Tampa.

[7] SHIRIAEv, A. S. and FradKov, A. L. (1998). Stabilization of invariant manifolds for nonlinear nonaffine systems, Proceedings of IFAC Conference NOLCOS'98, Enschede, pp. 215-220.

[8] Shiriaev, A. S., Ludvigsen, H. and Egeland, O. (1999). Swinging up of the spherical pendulum, Proceedings of the 14th IFAC World Congess, Beijing, pp. 65-70.

[9] Shiriaev, A. S., Ludvigsen, H., Egeland, O. and Fradkov, A.L. (1999). Swinging up of non-affine in control pendulum, Proceeding of American Control Conference, ACC'99, San Diego, pp. $4040-4044$. 